\title{
Distinct genital tract HIV-specific antibody profiles associated with tenofovir gel
}

This article has been corrected since Advance Online Publication and a erratum is also printed in this issue

D Archary ${ }^{1,11}$, KE Seaton ${ }^{2,11}$, JS Passmore ${ }^{1,3,4}$, L Werner $^{1}$, A Deal $^{2}$, LJ Dunphy $^{5}$, KB Arnold ${ }^{5}$, NL Yates ${ }^{2}$,

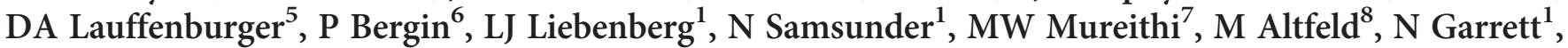
Q Abdool Karim ${ }^{1,9}$, SS Abdool Karim ${ }^{1,9}$, L Morris ${ }^{1,4}$ and GD Tomaras ${ }^{2,10}$

The impact of topical antiretrovirals for pre-exposure prophylaxis on humoral responses following HIV infection is unknown. Using a binding antibody multiplex assay, we investigated HIV-specific IgG and IgA responses to envelope glycoproteins, p24 Gag and p66, in the genital tract (GT) and plasma following HIV acquisition in women assigned to tenofovir gel $(n=24)$ and placebo gel $(n=24)$ in the CAPRISA 004 microbicide trial to assess if this topical antiretroviral had an impact on mucosal and systemic antibody responses. Linear mixed effect modeling and partial least squares discriminant analysis was used to identify multivariate antibody signatures associated with tenofovir use. There were significantly higher response rates to gp120 Env $(P=0.03)$, p24 $(P=0.002)$, and p66 $(P=0.009)$ in plasma and GT in women assigned to tenofovir than placebo gel at multiple time points post infection. Notably, p66 IgA titers in the GT and plasma were significantly higher in the tenofovir compared with the placebo arm $(P<0.05)$. Plasma titers for 9 of the 10 HIV-IgG specificities predicted GT levels. Taken together, these data suggest that humoral immune responses are increased in blood and GT of individuals who acquire HIV infection in the presence of tenofovir gel.

\section{INTRODUCTION}

Several recent HIV prevention trials have tested the efficacy of tenofovir containing pre-exposure prophylaxis (PrEP) regimens in oral ${ }^{1-3}$ or topical form, ${ }^{4}$ with protective effects ranging from 0 to $86 \% .{ }^{1-6}$ While poor adherence to PrEP has been identified as a major factor limiting efficacy in these trials, the observed disparity in protection urges further investigation into possible biological mechanisms associated with no to incomplete protection. Preclinical studies in non-human primates and in women in the CAPRISA 004 clinical trial have suggested that exposure to PrEP preserves the magnitude of HIV-1specific CD4 cell responses generated in those experiencing breakthrough HIV infections. ${ }^{7,8}$ Investigations of humoral immunity following breakthrough infections showed slower development of HIV-specific antibody avidity. ${ }^{9,10}$ In addition, decreased titers were shown in HIV-infected individuals on antiretroviral treatment. ${ }^{11-13}$ The effect of topical tenofovir on the magnitude and kinetics of mucosal and systemic antibody responses remains an important gap in our knowledge.

Antibody responses at the portal of HIV entry, the mucosa of the lower female reproductive tract, are thought to be a key mechanism to block virus dissemination from the GT and to prevent or delay replication and establishment of a productive infection. ${ }^{14-16}$ Vaccine-induced, locally produced

${ }^{1}$ Centre for the AIDS Program of Research in South Africa, University of KwaZulu-Natal, Durban, South Africa. ${ }^{2}$ Duke Human Vaccine Institute, Duke University Medical Center, Durham, North Carolina, USA. ${ }^{3}$ Institute of Infectious Diseases and Molecular Medicine, University of Cape Town, Cape Town, South Africa. ${ }^{4}$ National Institute for Communicable Diseases of the National Health Laboratory Services, Johannesburg, South Africa. ${ }^{5}$ Department of Biological Engineering, Massachusetts Institute of Technology, Cambridge, Massachusetts, USA. ${ }^{6}$ Imperial College, International AIDS Vaccine Initiative Core Immune Monitoring Laboratory, London, UK. ${ }^{7}$ KAVI Institute of Clinical Research, School of Medicine, College of Health Sciences, University of Nairobi, Nairobi, Kenya. ${ }^{8}$ Heinrich-Pette Institut, Leibniz Institute for Experimental Virology, University of Hamburg, Hamburg, Germany. ${ }^{9}$ Department of Epidemiology, Mailman School of Public Health, Columbia University, New York, NY, USA and ${ }^{10}$ Departments of Surgery, Immunology and Molecular Genetics and Microbiology, Duke University, Durham, North Carolina, USA. Correspondence: D Archary (desh.archary@caprisa.org) or G Tomaras (georgia.tomaras@duke.edu)

${ }^{11}$ These authors contributed equally to this work. 
gp41 SIV Env IgG in the female macaque GT correlated with protection in animals receiving a high-dose, intra-vaginal challenge 20 weeks post vaccination. ${ }^{16,17}$ In addition, in highlyexposed persistently seronegative (HESN) women, the presence of mucosal HIV-specific antibodies has been suggested to correlate with protection. ${ }^{18-21}$ We found gp120 specific IgAs but no HIV-1-specific IgG responses in GT fluid in HESN women recruited into the HPTN 035 microbicide trial. ${ }^{21}$ In HIV-infected women, we showed that both gp41- and gp120-specific IgA and IgG were detected in the GT. ${ }^{21,22}$

Given these findings, a major goal of HIV prevention research is to induce protective immunity at the genital mucosa. Defining the properties of the earliest antibody responses at the vaginal mucosa following HIV transmission will enable a better understanding of the potential role of tenofovir in modulating protective antibody responses in the female GT. We hypothesized that higher titer antibodies and increased breadth of HIVspecific antibody responses would be seen in plasma as well as in the GT of women enrolled in the CAPRISA 004 trial, because of the likely exposure to HIV in the GT in the presence of tenofovir. This is supported by previous observations of preserved HIV-specific CD4 cell responses in the tenofovir compared with the placebo arm. ${ }^{8}$ We compared HIV-1 antibody response rates and titers of IgG and IgA responses in plasma and cervicovaginal lavages (CVLs) with a panel of 10 HIV-specific antibody epitopes. Women in the tenofovir arm could be differentiated from the placebo arm by distinct HIV-1-specific antibody signatures, including plasma and CVL IgA responses to p66 during early HIV-1 infection. Elucidation of the effects of microbicides on HIV-1 antibody responses and evolution in those who become infected will assist in the design and development of future combination prevention strategies.

\section{RESULTS}

\section{Study participants}

Overall, 58.3\% (28/48) of the women were from rural areas in KwaZulu-Natal, with only $54.2 \%$ (26/48) completing high school and most in stable partnerships (Table 1). The median age of the women was 23 years, with a median blood CD 4 count of 498 cells per $\mu$ l during acute HIV infection and plasma viral load of 59,050 copies per $\mathrm{ml}$. No differences were found between the two arms for age, education, hormonal contraceptive use, marital status, number of sexual partners, or condom use. Women included in the study were matched for CD4 T-cell counts during acute HIV infection since differences in CD4 helper function may have confounded analysis of HIVantibody responses. There were no differences between the arms for either CD4 cells or viral loads over time (data not shown). After matching, the earliest samples collected from women in this sub-study in the TFV arm, had a significantly longer time from enrollment to acquisition of HIV-1 infection (12.8 months (interquantile range (IQR) 6.6-16.6 months)) than women in placebo arm (7.4 months (IQR 3.3-10.6 months), $P=0.02$ ).
High IgG response rates to multiple HIV antigens in the genital tract

We investigated mucosal IgG and IgA antibody responses (indicated as the number of women responding to any HIV antigen) in the female GT for potential differences in tenofovir vs. placebo-exposed women. In the GT, IgG rather than IgA responses, were more readily detected (Figure 1 and data not shown). The majority of women had GT IgG responses to multiple HIV-1 antigens, compared with more restricted GT IgA responses (Figure 1 and Supplementary Figure 1 online). All of the women had detectable GT IgG responses to p24, p66, gp140, and gp41 (Figure 1d,e and data not shown). In contrast, GT IgG responses to gp70 V1V2, Consensus Group M gp120, and V3 antigens were seen less frequently and emerged only later after infection (Figure 1a-c). There was no major difference noted between the study arms except for the rates of GT IgG responses to Clade CV3 linear epitopes were 1.7 times higher in the tenofovir $(88.2 \%)$ than placebo arm $(52.4 \%)$ at 12 months post infection $(P=0.03$, Figure $1 c)$.

While GT IgA responses to p66 and p24 were detected, gp70 V1V2 and Consensus Group M gp120-IgA responses were not detected (Figure 1f,g,i,j). Women in the tenofovir arm were 3.6 times more likely to have p66 IgA responses than those in the placebo arm (95\% confidence interval (CI) 1.38-9.61; $P=0.009$; Figure 1j). No further differences in GT IgA response rates were found between the arms.

\section{Higher prevalence of plasma IgA responses detected in tenofovir compared with placebo arm}

Plasma HIV-1-specific IgG and IgA response rates, indicating the number of women responding to any HIV antigen, were compared to determine whether differences existed between antibody emergence and specificity in those assigned to tenofovir or placebo (Figure 2). The majority of women had HIV-1-specific IgG responses in plasma that did not differ by treatment arm (Figure 2a-e). P24 and p66 were the dominant plasma HIV-1-specific IgG responses in the first 3 months after infection (Figure 2d,e), with $100 \%$ of women in both arms recognizing these antigens. Consensus Group M gp120 IgG responses in plasma emerged more slowly than p24 and p 66 responses, with only $35 \%$ of women being IgG positive for gp 120 at 3 months and $57 \%$ being positive at 6 months post infection (Figure 2b). Plasma IgG response rates to gp70 V1V2 were generally high throughout the course of infection, ranging from 73 to $100 \%$ response rate in both arms (Figure 2a). Notably, HIV-infected women from the tenofovir arm had $\sim 4$.2-fold higher response rates to gp70 V1V2 than those in the placebo arm (odds ratio $=0.8-21.195 \% \mathrm{CI}, P=0.08$ ), although this was not significant. In addition, women in the tenofovir arm were 1.8 times more likely to have a Consensus Group M gp120-IgA response from 6 to 12 months (95\% CI 1.06-3.11, $P=0.08$ ) (Figure 2g). Plasma IgA response rates to p24 (odds ratio $=3.64 ; 95 \%$ CI 1.49-6.38; $P=0.002$ ) and p66 (odds ratio $=2.92 ; 95 \%$ CI $1.68-5.07 ; \quad P=0.0001)$ were also significantly higher in the tenofovir than in the placebo arm (Figure 2i and $\mathbf{j}$ ). 
Table 1 Behavioral and clinical characteristics of the women in this study

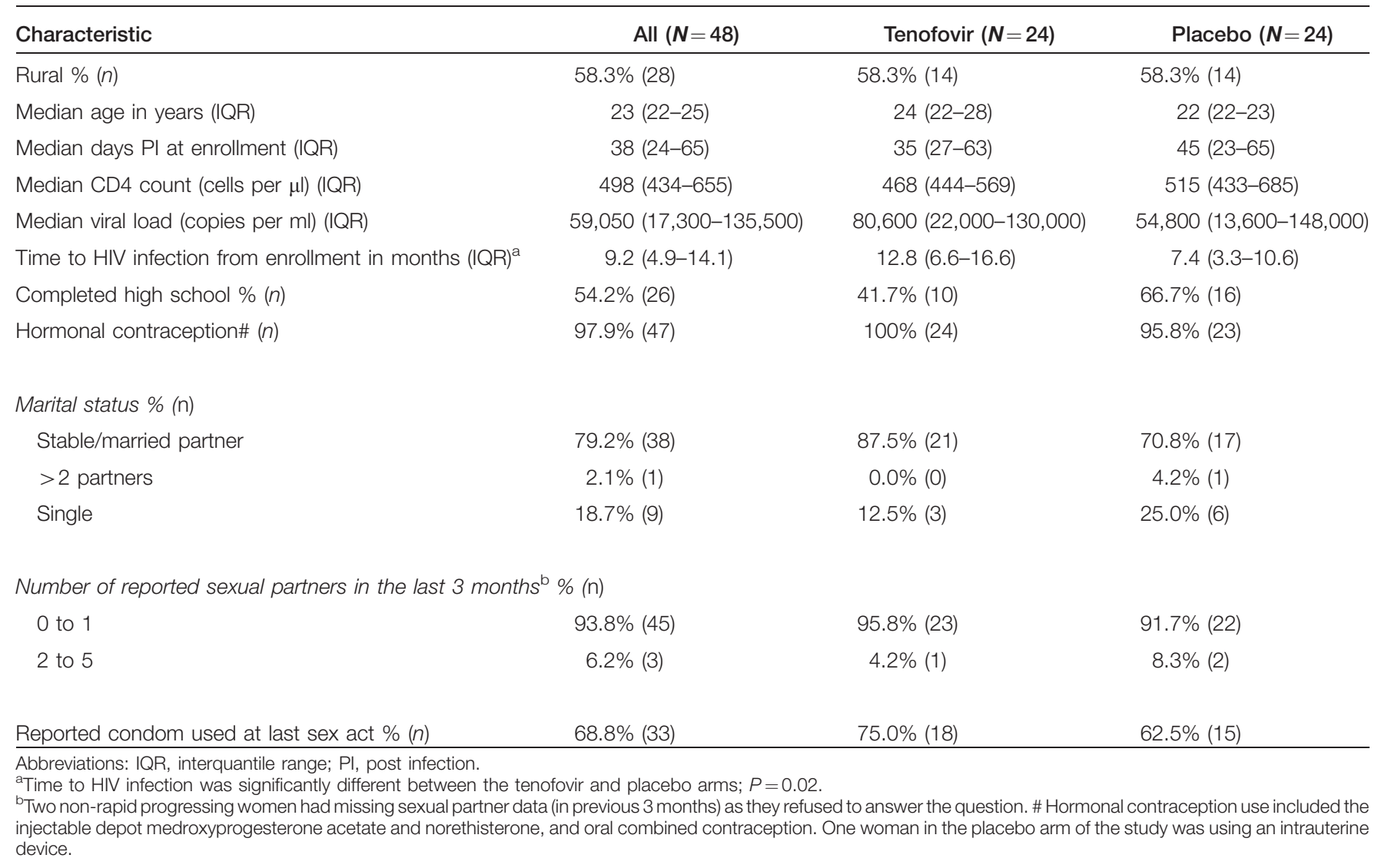

\section{Higher titers of IgG and IgA in plasma and GT in women in the tenofovir arm}

The magnitude of HIV-specific antibody responses, indicative of titers, in plasma and CVL of women in the tenofovir arm were then compared with women in the placebo arm. Similar titers of plasma HIV-specific IgGs were noted in the tenofovir and placebo arms for 9 out of 10 of the antigens tested (Supplementary Figure 2). Only IgG titers directed against gp70 V1V2 were significantly higher in plasma from women in tenofovir arm compared with women in the placebo arm in the first 6 months post infection $(P=0.009$; adjusted and data not shown). Plasma IgG responses were generally of similar magnitude between the placebo arm and tenofovir arm for most HIV-1 antigens (Figure 3a; Supplementary Figure 2). However, mean titers of plasma Consensus Group M gp120 $(P=0.05), \mathrm{p} 24$ and p66 IgA responses were higher in the tenofovir treatment arm than the placebo arm. Plasma p24 IgA titers were significantly higher over time and at $>6$ months post infection in the tenofovir-treated compared with the placebo arm $(P=0.016$ and $P=0.012$, respectively, Supplementary Figure 3) and p66 IgA titers at 6 months trended higher $(P=0.08)$ and was significantly different at 12 months $(P=0.004)$ post HIV infection (Figure 3b, Supplementary Figure 3), although not after adjusting for multiple comparisons. In addition, increased titers of p66 IgGs were found in CVL from tenofovir-treated women compared with those in the placebo arm 12 months $(P=0.04)$ post infection (Figure 3c; Supplementary Figure 4). This trend was also noted for IgA responses in the CVL (Supplementary Figure 5); although overall IgA response rates and magnitudes were lower in CVL and thus differences could not be compared between groups.

\section{GT and plasma p66 IgA responses differentiated women in the tenofovir arm}

A multivariate approach that integrates antibody measurements over time and takes tissue compartment into account was used to determine if there were any unique antibody signatures associated with tenofovir use. A feature reduction method (Least Absolute Shrinkage and Selection Operator (LASSO)) and partial least squares discriminant analysis (PLSDA) were used together to identify the minimum linear combination of antibody specificities that differentiated tenofovir and placebo groups, using 6 and 12 month post infection time points. This approach has been used previously to identify molecular signatures associated with disease status ${ }^{23}$ or cellular behaviors. ${ }^{24}$ LASSO identified a subset of $8 \mathrm{HIV}-1$ antibody specificities that best differentiated tenofovir and placebo groups, with $77 \%$ cross-validation accuracy and $87 \%$ calibration accuracy (Figure 4a,b). Five of these eight specificities were strongly and positively associated with the tenofovir arm (negatively loaded on LV1; including plasma p66 

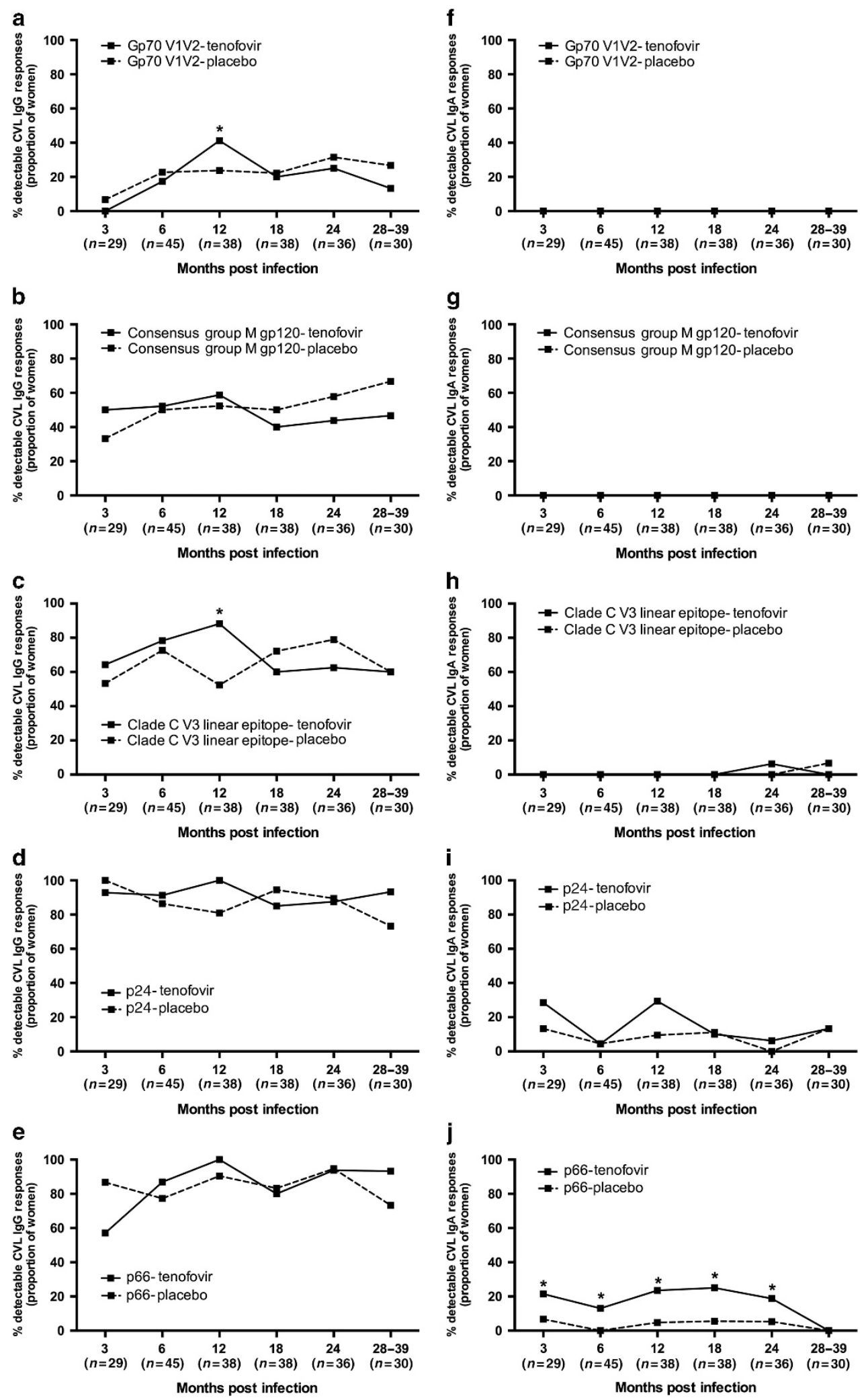

Figure 1 Mucosal HIV-1-specific IgG but fewer IgA responses in the female genital tract of HIV-1-infected women participating in the CAPRISA 004 microbicide trial. Cervicovaginal lavage HIV-1 IgG (a-e) and HIV-1 IgA (f-j) to gp70 V1V2, gp120, V3, Gag and p66 antigens were measured in HIV-1-infected CAPRISA 004 participants from +3 to 39 months post infection. Solid lines represent participants in the Tenofovir arm and dotted lines represent participants in the placebo arm. In parentheses below are the sample sizes for each time point. Asterisks indicate significant differences in detection between the arms at respective time points (Fisher's exact test). 

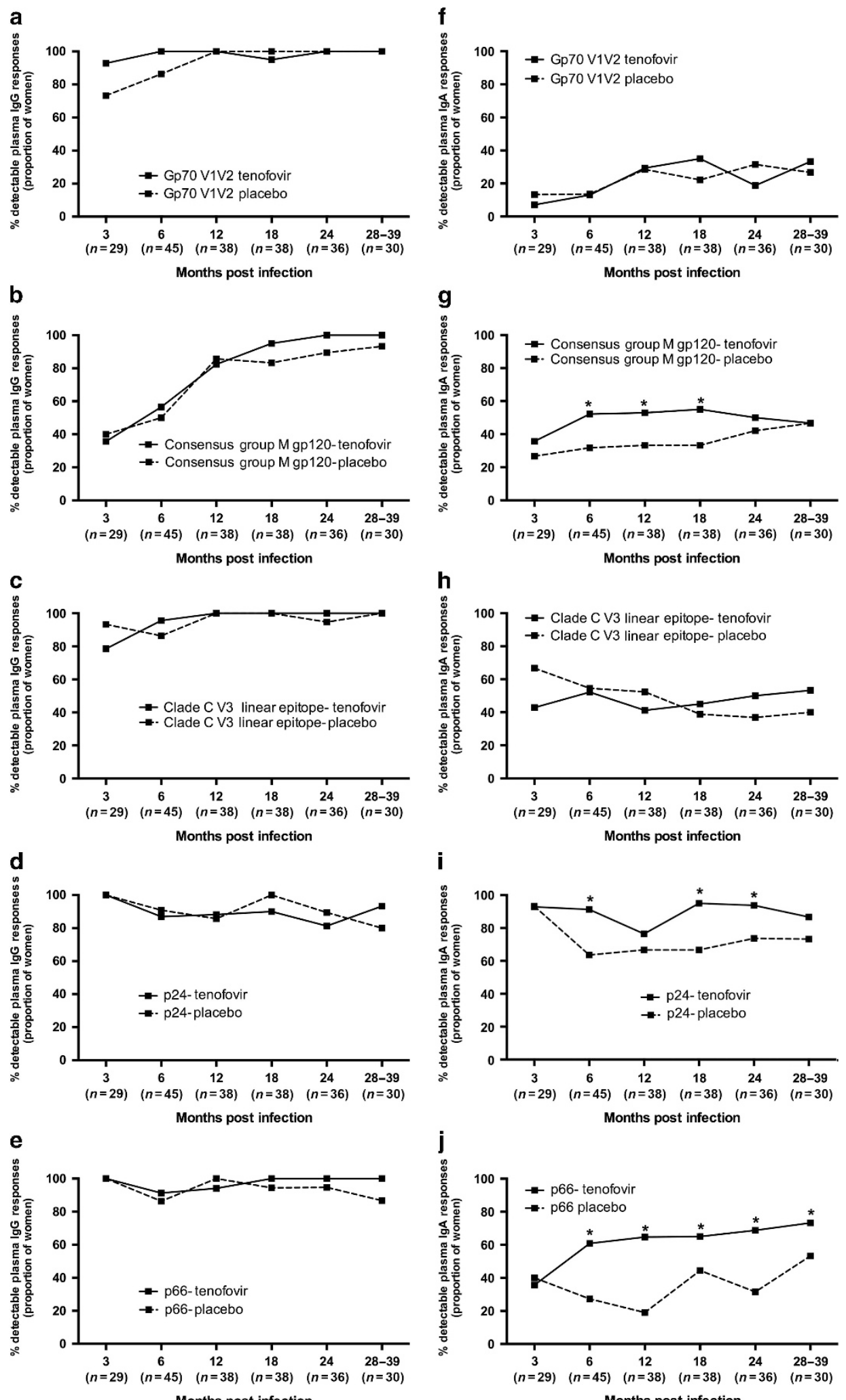

Figure 2 Plasma HIV-1-specific IgG and IgA are frequently detected in HIV-1-infected women participating in the CAPRISA 004 microbicide trial. Plasma HIV-1 IgG (a-e) and HIV-1 IgA (f-j) to gp70 V1V2, gp120, V3, Gag and p66 antigens were measured in HIV-1-infected CAPRISA 004 participants from +3 to 39 months post infection. Solid lines represent participants in the tenofovir arm and dotted lines represent participants in the placebo arm. In parentheses below are the sample sizes for each time point. Asterisks indicate significant differences in detection between the arms at respective time points (Fisher's exact test). 
a

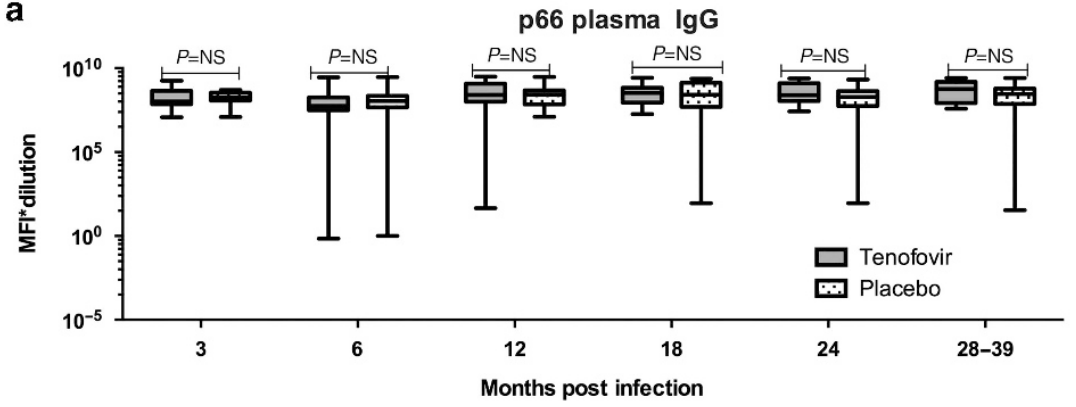

b
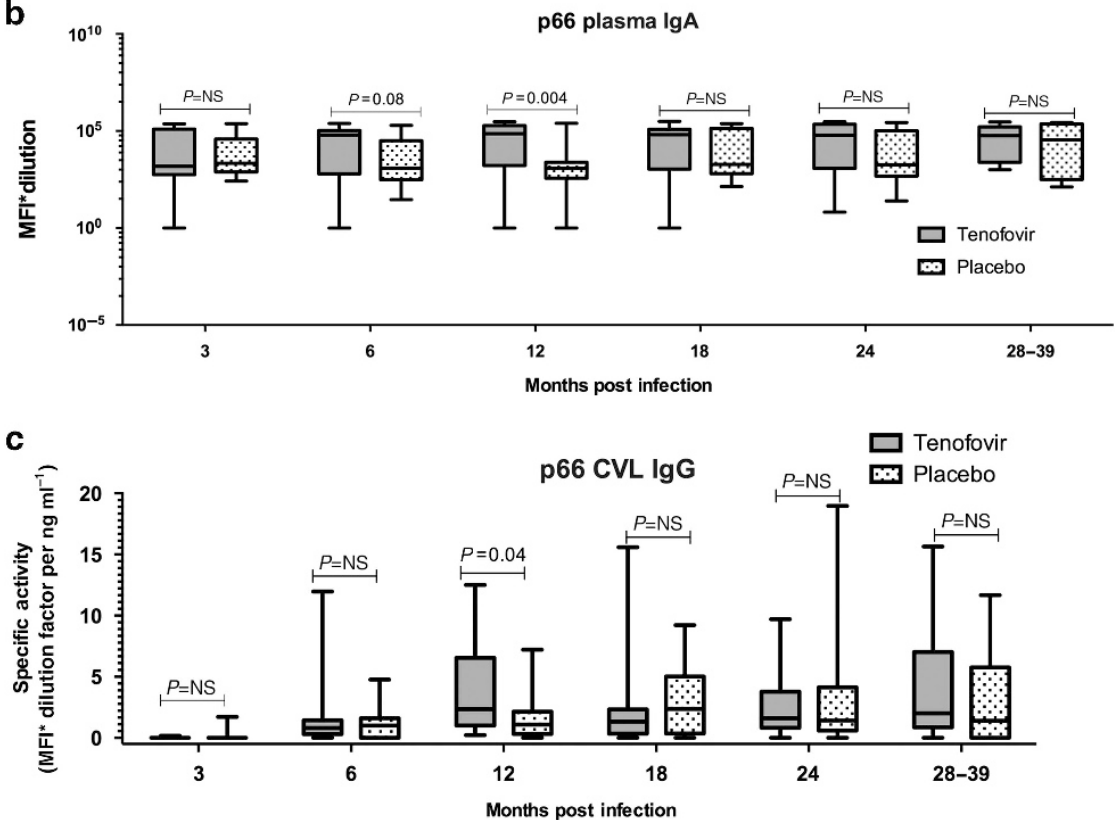

Figure 3 Increased p66 plasma IgA and CVL IgG response magnitude in acute infection with prior tenofovir use. Plasma response magnitude is presented as MFI*dilution in $\mathbf{a}(\mathrm{IgG})$ and $\mathbf{b}(\mathrm{IgA})$. CVL response magnitude is presented as specific activity $\left(\mathrm{MFl}^{*}\right.$ dilution factor per $\mathrm{ng} \mathrm{ml^{-1 }}$ ) in $\mathbf{c}(\mathrm{IgG})$. Boxes denote the 25th and 75 th percentile and whiskers denote the minimum and maximum values. The horizontal line represents the median response. Responses with $\mathrm{MFI}<100$ are below the limit of detection for this assay. $P<0.05$ were statistically significant. Comparisons of MFIs between tenofovir and placebo were done using the Wilcoxon Rank Sums test. CVL, cervicovaginal lavage; MFI, mean fluorescent intensity; NS, not significant.

IgA at months 6 and 12, CVL p66 IgA at month 6, plasma p66 IgG at month 12, and CVL gp41 IgA at month 12), while three out of eight were negatively associated with the tenofovir arm (positively loaded on LV1; including plasma Clade C V3 linear epitope IgA at month 12, p24 IgG at month 6, and Consensus Clade C Env gp 140-IgG at month 12) (Figure 4b). Confirmation of these identified signatures was done by generating 1,000 additional PLSDA models, each with 8 different combinations of antibody specificities selected from the remaining (non-LASSO) measurements. This analysis suggested that the LASSO-selected signature was significantly better for differentiating tenofovir and placebo groups $(P<0.01)$, as both calibration accuracy and cross-validation accuracy were in the 99th percentile rank compared with other models (Supplementary Figure 6A,B). The identified signature suggested spatio-temporal relationships between HIV-specific antibody specificities that differentiated women in the tenofovir from placebo arm. Earlyand late-antibody relationships between $\mathrm{p} 66$ in plasma and CVL contributed most notably to this signature, with plasma p66 IgA (months 6 and 12), CVL p66 IgA, and IgG (months 6 and 12, respectively) strongly negatively loaded onto LV1, and strongly associated with the tenofovir arm (the more negative the LV1 loading, the more strong the association is for the tenofovir arm) (Figure 4b). This suggested that women in the tenofovir group were best characterized by elevated p66 IgA responses over time in both plasma and CVL compared with the placebo group. In addition, lower IgA responses to Clade C V3 linear epitope (month 12), IgG to p24 (month 6), and IgG to Consensus Clade C Env gp140 (month 12), all positively loaded on LV1 and detected in plasma, best characterized women in the tenofovir arm from women in the placebo group (the more positive the LV1 loading, the stronger the association is for the placebo arm). These results support findings from the univariate analysis, and provide complementary insight into possible integrated relationships between different antibody specificities and provide further evidence of a detectable tenofovir effect on humoral immunity in women subsequent to seroconversion. 
a
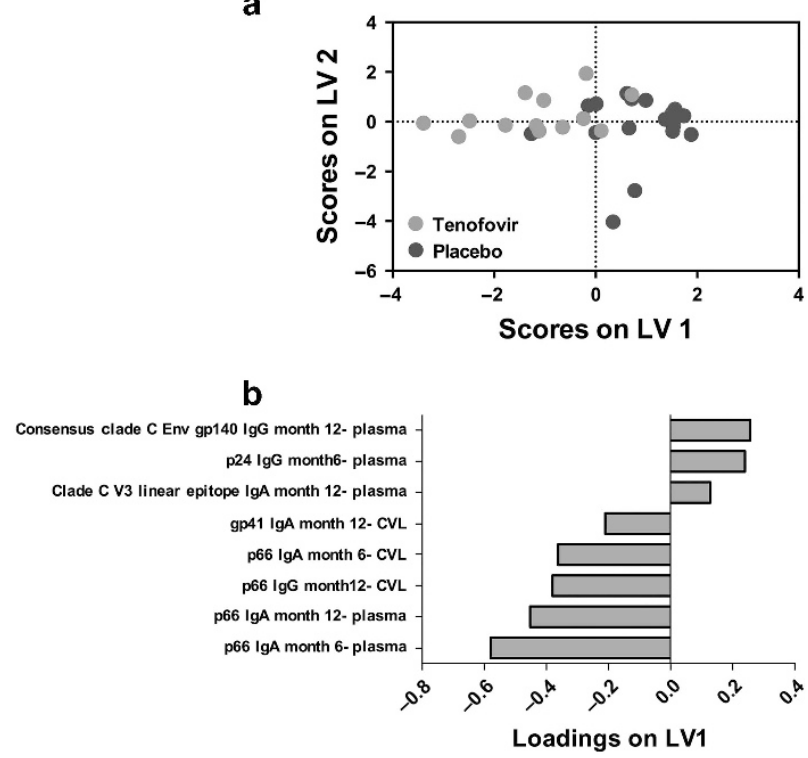

Figure 4 Six- and 12-month antibody signatures associated with participants in the tenofovir and placebo arms. LASSO identified a signature of eight antibody specificities that separated tenofovir (red $n=13$ ) and control groups (blue $n=18$ ) in a PLSDA model with $83 \%$ calibration accuracy and $77 \%$ cross-validation accuracy (scores plot; a). The loadings plot depicts weighted loadings of individual antibody specificities within the distinguishing signature (b). Five of these specificities were positively associated with tenofovir (negatively loaded on LV1; plasma p66 IgA at months 6 and 12, plasma p66 IgG at month 12, CVL p66 IgA at month 6, and CVL gp41 IgA at month 12) and three were negatively associated with tenofovir (positively loaded on LV1; plasma Clade C V3 linear epitope IgA at month 12, p24 IgG at month 6 and Consensus Clade C Env gp140-lgG at month 12). Latent variable 1 accounted for $24.84 \%$ and latent variable 2 accounted for $15.38 \%$ of data variance. For the analysis, only 6- and 12-month measurements were used for participants where $<4 \%$ of the total data was missing. CVL, cervicovaginal lavage.

\section{Compartmental associations for Gag p24 IgGs from women in the tenofovir arm}

To determine whether HIV-1-specific mucosal antibodies were locally produced or predominantly transudated from plasma, we compared the titers of HIV-specific IgG and IgA in plasma with those in the CVL. Among women assigned to the tenofovir arm, the plasma p24 IgG responses correlated significantly to those detected in matching CVL, at several time points post infection $(6$ months $(\beta$-coefficient $=1.4, P<0.0001)$ until $>30$ months $(\beta$-coefficient $=1.3, P<0.0001))($ Figure 5a). In addition, Consensus Clade C Env gp140 and Group M consensus gp140 Env IgG responses at 6 months post infection in the tenofovir group correlated significantly between the compartments (Figure 5a). P24 IgG responses in the placebo group showed more restricted and less strong associations between plasma and CVL (Figure $\mathbf{5 b}$ ).

No correlation was seen between IgA responses in the plasma and CVL at any time point for either arm, which were detected at lower titers in both compartments (Figure 5c, d). This suggests that HIV-1-specific IgAs are predominantly locally produced in the female GT although absolute antibody titers may have influenced this poor concordance.

\section{Plasma viral load correlated with HIV-specific IgG and IgA responses}

We evaluated whether plasma viral loads were associated with titers of HIV-specific IgGs and IgAs in the plasma. HIV-specific plasma IgG titers correlated significantly with viral load (Table 2). In the tenofovir arm, titers of IgG directed against 1086 C.gp140, Consensus Clade B gp41 ID epitope, and gp41 correlated with plasma viral load, although not after adjusting for multiple comparisons. For HIV-1-specific IgA responses (Table 2), plasma viral load correlated positively with several plasma IgA specificities from women in the placebo arm (clade C V3 linear epitope, Group M consensus gp140 Env, and consensus clade C gp140).

Similarly, we found broadly negative associations between CD4 cells and HIV-specific IgG responses to several antigens in women in the tenofovir arm at 6 months post infection, suggesting that HIV clinical status was influencing HIVspecific antibody responses (Table 3; for Consensus Group M gp120 $(r=-0.49, P=0.02), 1086$ C.gp140-IgG $(r=-0.53$, $P=0.01)$, Consensus Clade C Env gp140-IgG $(r=-0.48$, $P=0.02$ ); although not significant after false discovery rate adjustment). There were no associations with $\mathrm{CD} 4$ cells and IgG responses in the placebo arm.

For HIV-1-specific IgA responses, viral loads correlated positively in the placebo arm for 8 out of 10 of the IgA antigen specificities (Table 2; Clade C V3 Linear epitope, Group M consensus gp140 Env -IgG, Consensus Clade C Env gp140 remained significant after adjusting for false discovery rate). Similarly, in the tenofovir arm at 6 months post infection, we found a significant negative association with $\mathrm{CD} 4$ cells and HIV-specific IgA responses (Table 3; including 1086 C.gp140IgG, Consensus Group M gp140, and Consensus Clade C Env gp140). These data indicate that both IgG and IgA responses may be driven by viral load, which can negatively impact CD 4 cells. However, other factors such as characteristics of the transmitted virus, pre-existing immune responses, concurrent infections, and host genetics may also contribute.

\section{DISCUSSION}

Women assigned to the tenofovir gel had a distinct p66 IgA signature in both the GT and plasma that differentiated them from those in the placebo arm following HIV-1 infection. The enhanced p66 IgA responses over time in both compartments with a parallel delay or lowering of IgA- specific V3, and IgGspecific Env gp140 responses indicates the impact of prior topical tenofovir on ensuing antibody responses post HIV-1 infection.

An increased frequency and magnitude of HIV-1-specific IgA responses to p66 and p24 in the plasma and GT were seen during the primary infection stage in the tenofovir compared with the placebo arm. Previous studies have shown that IgG rather than IgA are predominant in the type II mucosa of the lower female genital mucosa. ${ }^{25,26}$ However, we previously demonstrated that altered antibody isotypes and specificities were present in CVLs of HESN women participating in the HPTN 035 microbicide trial (randomized to either BufferGel or $0.5 \%$ PRO2000 or placebo) compared with chronically 
a

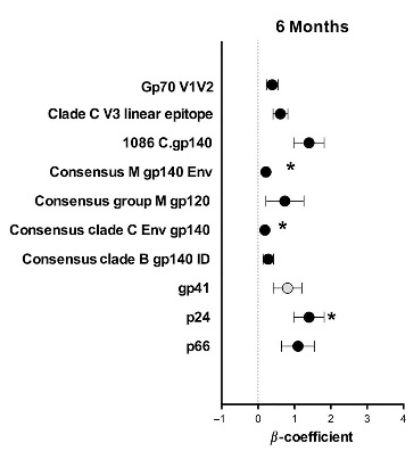

b

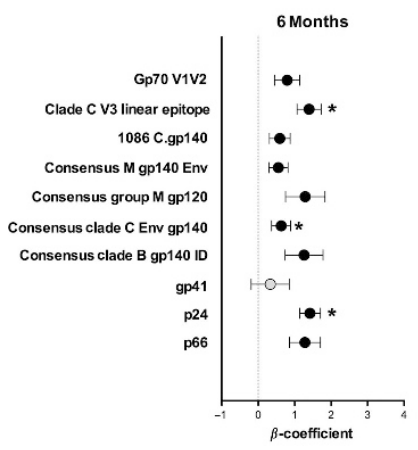

c

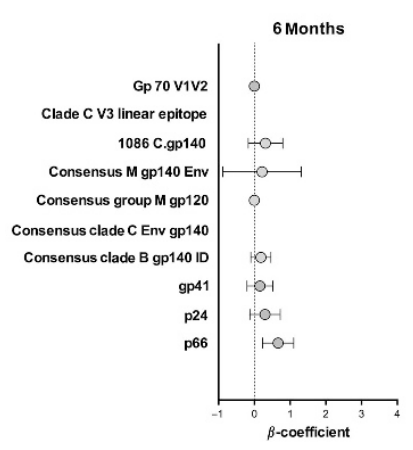

d

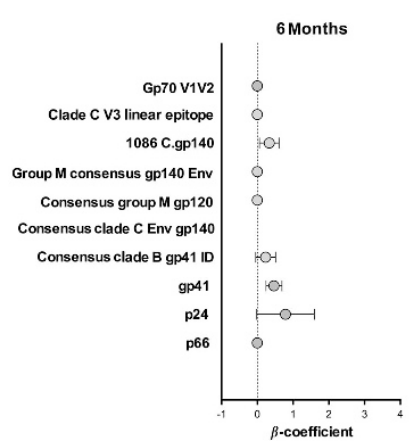

IgG plasma vs.CVL- tenofovir
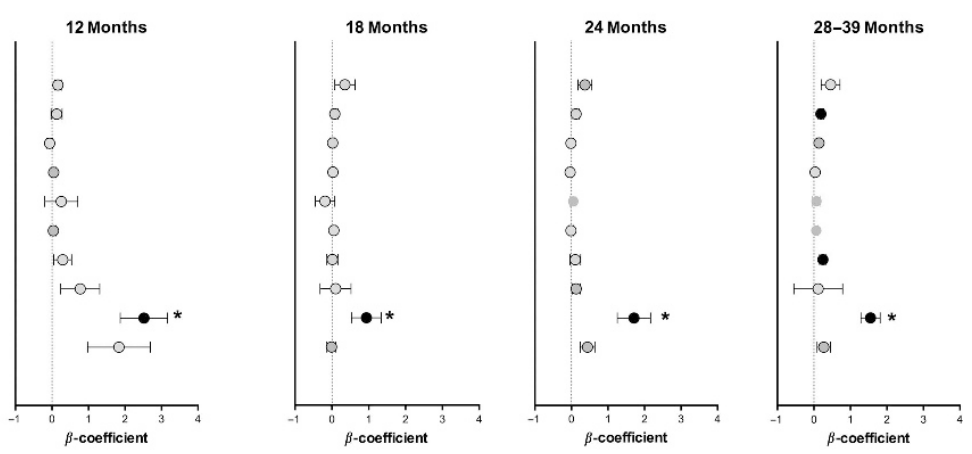

\section{IgG plasma vs.CVL- placebo}
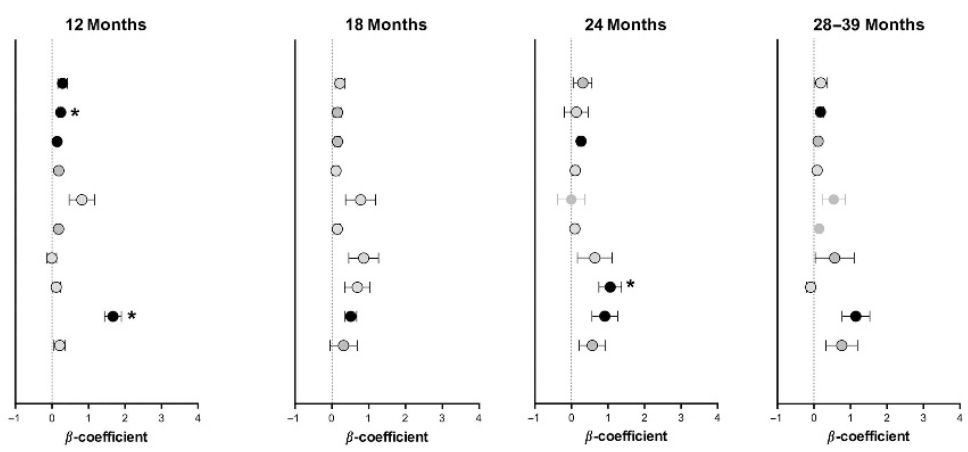

\section{IgA Plasma vs. CVL- Tenofovir}
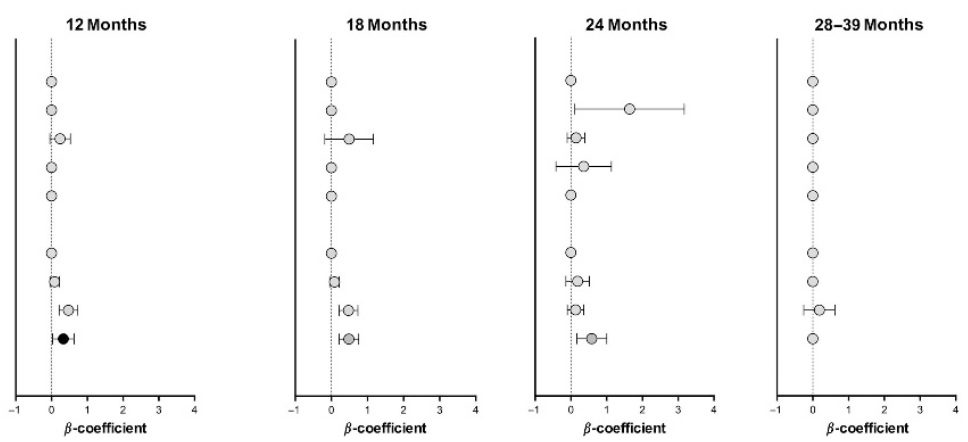

\section{IgA Plasma vs.CVL- Placebo}
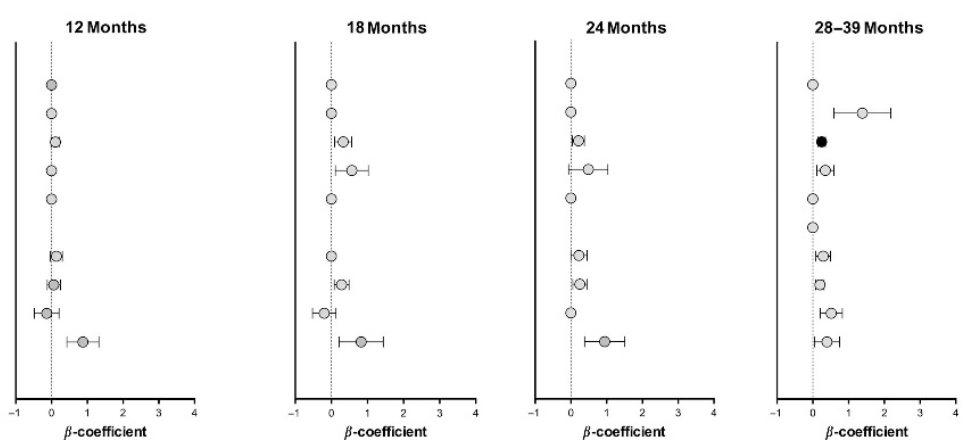

Figure 5 p24-specific IgG responses correlate between plasma and CVL in tenofovir-treated women. Beta ( $\beta)$ coefficients representing correlation between the magnitude of HIV-1-specific responses in plasma and CVL from +3 to 39 months post HIV-1 infection. IgG (a) and IgA (c) shown for the tenofovir arm and $\operatorname{lgG}(\mathbf{b})$ and $\operatorname{lgA}(\mathbf{d})$ are shown for the placebo arm. The colored circle $(\bullet)$ indicates a statistically significant correlation $(P<0.05)(\mathrm{F}$-test from Linear Mixed Model). An asterisk $\left(^{*}\right)$ denotes statistical significance adjusted for false discovery rate. CVL, cervicovaginal lavage. 
Table 2 Viral loads correlated with plasma $\lg G$ and $\lg A$ at 6 months in the tenofovir and placebo arms

\begin{tabular}{|c|c|c|c|c|c|c|c|c|}
\hline HIV antigen & $r$-value & $\boldsymbol{P}$-value & $r$-value & $\boldsymbol{P}$-value & $r$-value & $\boldsymbol{P}$-value & $r$-value & $\boldsymbol{P}$-value \\
\hline Clade C V3 linear epitope & 0.02 & NS & 0.58 & $0.003^{\mathrm{a}}$ & -0.1 & NS & 0.59 & $0.003^{\mathrm{a}}$ \\
\hline Consensus group M gp120 & 0.41 & 0.05 & 0.55 & $0.007^{\mathrm{a}}$ & 0.08 & NS & 0.47 & 0.03 \\
\hline Consensus group M gp140 & 0.23 & NS & 0.43 & $0.04^{a}$ & 0.06 & NS & 0.53 & $0.009^{a}$ \\
\hline Consensus clade C Env gp140 & 0.35 & 0.09 & 0.44 & $0.03^{\mathrm{a}}$ & 0.19 & NS & 0.53 & $0.009^{a}$ \\
\hline Consensus clade B gp41 ID epitope & 0.48 & 0.02 & 0.45 & $0.03^{a}$ & -0.08 & NS & 0.33 & NS \\
\hline Gp41 & 0.43 & 0.03 & 0.49 & $0.02^{\mathrm{a}}$ & 0.07 & NS & 0.46 & 0.02 \\
\hline p66 & 0.02 & NS & 0.73 & $<0.0001^{a}$ & 0.41 & 0.05 & 0.38 & 0.07 \\
\hline
\end{tabular}

Abbreviation: NS, not significant.

$P<0.05$ indicates a statistically significant correlation

aRemains significant after false discovery rate.

Table 3 CD4 cells correlated with plasma IgG and IgA at 6 months in the tenofovir and placebo arms

\begin{tabular}{|c|c|c|c|c|c|c|c|c|}
\hline HIV antigen & $r$-value & $\boldsymbol{P}$-value & $r$-value & $\boldsymbol{P}$-value & $r$-value & $\boldsymbol{P}$-value & $r$-value & $\boldsymbol{P}$-value \\
\hline Clade C V3 Linear Epitope & -0.18 & NS & -0.30 & NS & -0.18 & NS & -0.53 & $0.008^{a}$ \\
\hline Consensus Group M gp120 & -0.49 & 0.02 & -0.28 & NS & -0.48 & 0.02 & -0.47 & 0.02 \\
\hline Consensus Group M gp140 & -0.25 & NS & -0.25 & NS & -0.61 & $0.002^{\mathrm{a}}$ & -0.51 & 0.01 \\
\hline Consensus Clade C Env gp140 & -0.48 & 0.02 & -0.26 & NS & -0.77 & $<0.001^{\mathrm{a}}$ & -0.36 & NS \\
\hline Consensus Clade B gp41 ID epitope & -0.42 & 0.05 & -0.09 & NS & -0.37 & NS & -0.28 & NS \\
\hline Gp41 & -0.40 & 0.06 & -0.05 & NS & 0.42 & 0.05 & -0.42 & 0.05 \\
\hline p66 & -0.03 & NS & -0.24 & NS & -0.43 & 0.05 & -0.50 & 0.02 \\
\hline
\end{tabular}

Abbreviation: NS, not significant.

$P<0.05$ indicates a statistically significant correlation.

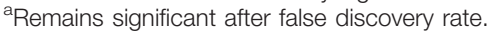

HIV-infected women. ${ }^{21}$ HIV-exposed-uninfected women in this trial exhibited an increase in different types of Env-specific IgA in the female GT compared with a HIV-1-infected cohort. In this sub-study of the CAPRISA 004 trial, women in the tenofovir arm had a delay of $>5$ months from enrollment to infection vs. women in the placebo arm. The p66 IgA signature in plasma and GT in tenofovir-exposed women may be a consequence of frequent exposure to HIV during sexual intercourse, without productive infection because of the presence of tenofovir, similar to the enhanced IgA responses seen in other HESN cohorts. ${ }^{14,21,27}$ Interestingly, prior studies have suggested that $\mathrm{p} 66$ antibodies are associated with the rate of HIV disease progression, ${ }^{28}$ in addition to being associated with HIV-1 controller status. ${ }^{29}$ Thus the role of p66 antibody in our study could also be a surrogate for another immune mechanism that is impacted by tenofovir use.
The significant delay in time to HIV infection in the presence of tenofovir may likely have played an indirect role in modulating specific antibody responses in the absence of HIV infection, as significant differences in p24- and p66-binding antibody response rates and magnitude were seen $>6$ months post infection. A limitation of this study is that samples immediately prior to infection or during very early acute infection were not available for investigation. Oral PrEP trials have shown immunomodulatory effects of tenofovir in those who became infected, with effects ranging from preserved CD4 cells to lower levels of systemic inflammation. ${ }^{30}$ In topical PrEP studies, Gag-specific T-cell responses in the absence of infection has been demonstrated $^{7}$ with use of tenofovir gel. During the acute stage of breakthrough infections in the CAPRISA 004 study, Laeyendecker et al. ${ }^{9,10}$ showed a delay in avidity maturation of specific antibody responses in women assigned to the tenofovir gel. 
While studies showed decreased antibody titers with ARVtreatment in individuals already infected with HIV $-1,{ }^{11-13}$ we found increased antibody response rates and magnitudes for V1V2 IgG-, p66- and p24-specific IgA and IgG during HIV-1 breakthrough infection in individuals exposed to tenofovir before infection after controlling for sexually transmitted diseases. Women with breakthrough infections in the tenofovir arm were reported to have an episodic use of 3-4 weeks of tenofovir gel post infection. ${ }^{4}$ In addition, high levels $(3.1 \times$ $10^{6} \mathrm{ng} \mathrm{ml}^{-1}$ ) of tenofovir-diphopshate were achieved in the genital tract with topical application. ${ }^{31}$ The half-life of tenofovirdiphopshate (active metabolite) is $\sim 40$ days in the genital tract, ${ }^{32}$ suggesting that the levels of TFV at mucosal sites were maintained for at least 6 weeks or 40 days after the last application of the tenofovir gel and this may explain the delay in antibody responses in the first few months post infection. Our study is different from the study by Laeyendecker et al. ${ }^{10}$ in that their study measured antibody avidity at early time points. Here, we measured the magnitude and breadth of the antibody response, not the avidity, to both envelope and non-envelope HIV-1 antigens. These data suggest that prior tenofovir use may prime the immune system ${ }^{7,8}$ on exposure to HIV, ${ }^{33}$ leading to increased p66- and p24-specific IgA and IgG that can distinguish patients with mucosally applied tenofovir during the primary stage of HIV infection.

In the RV144 vaccine trial, plasma gp70 V1V2-binding IgGs, including V1V2-specific IgG3, correlated with decreased HIV-1 infection risk. ${ }^{34-36}$ Whether the same specificity was present in the GT of uninfected vaccinees was not evaluated in the trial. Transudation of HIV-1-specific antibodies from the systemic circulation into secretions in the lower female GT has been described. ${ }^{37}$ Many factors may affect the transudation of antibodies across the mucosa, including vaginal $\mathrm{pH}^{38,39}$ the type of antibody glycosylation, ${ }^{40}$ and heterogeneity among individuals. Our data indicated that in the presence or absence of tenofovir, gp70 V1V2 IgG were detected in the GT in a small proportion of women throughout infection $(26 \%$ in the tenofovir and $22 \%$ in the placebo arm overall at 28-39 months), despite $100 \%$ of the women displaying plasma gp70 V1V2 IgG specificity (100\% for both tenofovir and placebo at 28-39 months). This indicates that there may be differential transudation of certain IgG specificities which are regulated by mechanisms that are yet to be determined. Whether these antibodies confer protection in the GT and whether tenofovir impacts the IgG subclass profile, ${ }^{34}$ requires further study. Observations for little to no gp70 V1V2-specific IgA in either the plasma or CVL suggests that certain antigen specificities may be less likely to have a mucosal IgA response. Although we did not measure the secretory component of IgA in this study (another limitation of this study), our data suggests that IgA responses may be generated both locally in the GT and systemically. Additional studies are needed to determine the relative proportion of locally produced vs. systemic IgA responses in the female GT, and whether HIV-1-specific IgA in different compartments may indeed protect or increase the risk for HIV acquisition. ${ }^{35,41,42}$ The menstrual cycle and female hormones may influence the composition of mucosal IgGs and possibly IgAs. We were unable to assess this in the current study as all women were on some form of hormonal contraception, and stage of the menstrual cycle in those cycling was not recorded.

The direct relationship between plasma viral loads and the magnitude of Env-specific IgGs suggested that B-cell immune responses may have been driven primarily through frequent antigen stimulation or exposure. ${ }^{43}$ IgG responses were found to be locally produced in the macaque GT, ${ }^{17}$ after SIVmac239 $\Delta$ nef vaccination and in response to live SIV exposure. Another study demonstrated that rectal Env-specific IgG responses were a key correlate of protection following heterologous prime boost vaccination. ${ }^{44}$ In the gut lamina propria of mice, local B-cell receptor editing, IgG repertoire, and B-cell development were shown to be regulated by extracellular signals from commensal microbes, ${ }^{45}$ and the same could be true for the female GT. ${ }^{46}$ The strong and significant cross-compartment correlations for most of the Env-specific IgGs may be reflective of local T-cell specificities in the GT. To achieve sterilizing protection against HIV, a combination of potent polyfunctional humoral immune responses that reach the GT and circulating T-cell-mediated immunity may be crucial. Whether HIVspecific antibodies in the GT would confer protection through combined direct neutralization and Fc-associated effector functions remains unknown. ${ }^{4-53}$

In summary, we identified specific alterations in the plasma and mucosal responses to HIV-1 infection following tenofovir gel use in those women who became infected. Our analyses revealed an antibody signature that distinguished women in the tenofovir arm post infection from those in the placebo arm. Further work is needed to evaluate whether tenofovir has a direct or indirect immuno-modulatory role that resulted in these differences in the humoral response to infection. We have corroborated earlier findings that systemic HIV-1-specific IgG responses likely transudate into the $\mathrm{GT}^{54,55}$ and that the IgA responses may be locally produced. These findings underscore the importance of both locally produced or transduced antibody responses in the genital tract to prevent infection. ${ }^{41,42,56-58}$ Information from this study is directly relevant to both vaccine and PrEP strategies, which aim to induce protective antibodies at mucosal surfaces.

\section{METHODS}

Study population and specimen collection. The University of KwaZulu-Natal's Biomedical Research Ethics Committee (E111/06), Family Health International's Protection of Human Subjects Committee (\#9946), and the South African Medicines Control Council (\#20060835) approved the CAPRISA 004 microbicide gel trial (Clinical Trials Number 00441298). Results of the primary analyses have been published previously. ${ }^{4}$ Written informed consent was obtained from all participants. This study was approved by the Institutional Review Board of Duke University and the University of KwaZulu-Natal's Biomedical Research Ethics Committee.

Forty eight women who seroconverted during the CAPRISA 004 1\% tenofovir microbicide gel trial were included in this study. ${ }^{4}$ Of these, 24 out of 48 were from the tenofovir arm (cases) and 24 out of 48 were from the placebo arm (controls), with cases and controls matched 
according to viral loads and CD4 T-cell counts at 6 months post infection. Recent HIV-1 infection was diagnosed on the basis of two HIV-1 rapid antibody tests and PCR (Roche Amplicor v1.5, Branchburg, NJ), and confirmed by enzyme immunoassay. Women were enrolled within 3 months of infection and the timing of infection was estimated either as the midpoint between the last HIV-1-negative test and the first antibody-positive test, or as 14 days before the first PCR positive test for antibody-negative participants. CD4 T-cell counts were assessed using a FACSCalibur flow cytometer, and viral loads were measured using a Cobas Amplicor HIV-1 Monitor Test, v1.5 (Roche Diagnostics, Branchburg, NJ). HIV-infected women were followed for a median of 36 months after enrollment (range 11.5-53.5 months), and plasma and CVL samples from 3, 6, 12, 18, 24, and $>30$ months post infection were included in this study. All women were naive to antiretroviral treatment for the duration of this sub-study. Plasma collected in EDTA was stored at $-80^{\circ} \mathrm{C}$ until use. CVL was collected from each woman at each study visit under speculum examination using $10 \mathrm{ml}$ sterile saline according to the method described by Bebell et al. ${ }^{59}$

HIV-specific binding antibody assay. HIV-specific antibodies against HIV-1-specific Env and Gag proteins were measured in plasma $(1: 50 ; 1: 10,000$, and $1: 100,000$ dilutions) and CVL (1:3 and 1:10 dilutions) using a customized HIV-1-binding antibody multiplex assay. ${ }^{22,34,35,60,61}$ IgG was depleted prior to performing HIV-1-specific IgA assays. ${ }^{22}$ The antigen panel included the following Env proteins: gp70_B.CaseA_V1_V2 (gp70 V1V2), Transmitted Founder Clade C gp140 (1086 C.gp140), ${ }^{62,63}$ C.con.env03 140 CF (Consensus Clade C Env gp140), Con6 gp120/B (Consensus Group M gp120), and Con S gp140 CFI (Group M consensus gp140 Env), and were provided by Drs Haynes and Liao, Duke Human Vaccine Institute, Durham, NC. ${ }^{64-66}$ Commercially available reagents included: p24 Gag and p66 RT (Protein Sciences), and gp41 (ImmunoDiagnostics). Consensus Clade B gp41 immunodominant (ID) epitope in tetramer form was kindly provided by Dr MA Moody, ${ }^{67}$ Duke Human Vaccine Institute). The sequences of the immunodominant epitope and the $\mathrm{V} 3$ peptide are as follows: (Biotin CRVLAVERYLRDQQLLGIWGCSGKLICTTAVP WNASWSNKSLNKI). Bio-V3.C (Clade C V3 linear epitope, Biotin CRVLAVERYLRDQQLLGIWGCSGKLICTTAV from JPT Peptide Technologies, Berlin, Germany).

All assays were run under good clinical laboratory practice-compliant conditions, including tracking of positive controls by Levey-Jennings charts. Positivity cut-offs for binding antibody responses in plasma for antibody-antigen pairs were pre-determined from $30 \mathrm{HIV}$-1-negative individuals (mean fluorescent intensity (MFI) +3 s.d.). Positive controls included titrations of $\mathrm{HIV}-1^{+}$purified IgG (HIVIG), CH58 IgG mAb, and 7B2 IgG or IgA mAbs (mAbs from Drs Liao and Haynes).

Total immunoglobulin quantification and HIV-SA in CVL. Total IgG and IgA in CVL were quantified using a 7-plex Bio-Rad Human Isotyping kit on the Bio-Plex 200 multiplex system (Bio-Rad, Hercules, $\mathrm{CA})$, according to the manufacturer's instructions. Ig concentrations were determined by 4-PL logistic regression using the Bio-plex Manager 6.0 software (Bio-Rad). HIV-1-specific activity (SA) was defined as the antigen-specific MFI (adjusted for dilution factor) divided by the total immunoglobulin amount (antigen-specific $\mathrm{MFI}^{\star}$ dilution per $\mathrm{ng} \mathrm{ml}^{-1}$ total IgG or IgA) to adjust for individual variation in total Ig recovered when performing CVL. HIV-1 antibody responses were considered positive if they met both antigen-specific positivity criteria and SA criteria (mean SA +3 s.d.) from a set of 30 seronegative CVLs. Samples that did not meet the positivity cutoff for SA were set to $1 / 10$ of the SA cut-off for statistical analysis and visualization purposes.

Statistical analysis. Fisher's exact test was used to compare the proportion of detectable IgGs at a single time point between the two arms. Linear mixed models were used to assess the impact of CAPRISA 004 gel arm assignment on both plasma and GT IgG and IgA measurements post HIV infection. Linear mixed models were also used to measure the effect of the plasma IgG levels on that of the GT at the same time point, and the effect of CD 4 cells and HIV-1 viral load on IgG levels within each arm. $\beta$-coefficients were reported as per 50 cells per $\mu$ increase in CD4 cells and per 1 log increase in plasma viral load. Odds ratios were determined by fitting a generalized estimating equation model with a binomial distribution. IgG values were log transformed to ensure normality and all $P<0.05$ were considered statistically significant. The false discovery rate method (BenjaminiHochberg) was used to adjust $P$ values for multiple comparisons to prevent a Type 1 error and circumvents over estimating statistical significance. Statistical analysis was performed using SAS version 9.3 (SAS Institute, Cary, NC).

Identification of antibody signatures with PLSDA. Partial least squares discriminant analysis (PLSDA) and the LASSO method for regression shrinkage and selection ${ }^{68,69}$ were used to identify antibody signatures across time points, and in plasma and CVL compartments, that best differentiated women in the placebo arm from those in the tenofovir arm. Due to missing measurements for some time points, only measurements made at 6 and 12 months were included in this analysis. After eliminating individuals with missing measurements, 31 out of 48 women were included (18 placebo; 13 tenofovir). All analyses were performed using Matlab software (MathWorks, Natick, MA) and PLS_Toolbox for Matlab (Eigenvector Research Incorporated, Wenatchee, WA). LASSO was implemented using K-fold crossvalidation to identify the model with the lowest possible mean squared error for prediction and minimum set of antibodies. PLSDA was used to evaluate the classification accuracy of the selected group of antibodies and was compared with classification obtained from individual measurements alone. Loading values indicate the degree of separation of antibody measurements between the tenofovir and placebo arms. Positive or negative loading values on latent variables identified by the PLSDA model were used to indicate antibody signatures that best separate tenofovir and placebo arms. The more positive or the more negative a loading on latent variables in the model, the more strongly that feature contributes to the distinguishing profile. Score plots were used to illustrate the ability of these signatures to distinguish tenofovir and placebo groups. Cross-validation was performed by repeatedly excluding contiguous subsets of data (five to six data points per set) during model calibration. Excluded data were then used to test model predictions. Raw data were normalized with mean centering and variance scaling prior to analysis.

SUPPLEMENTARY MATERIAL is linked to the online version of the paper at http://www.nature.com/mi

\section{ACKNOWLEDGMENTS}

We thank all the CAPRISA 002 Acute Infection Study participants who are continuing to make an important contribution to HIV research. The scientific and supportive role of the whole CAPRISA 002 study and protocol teams are gratefully acknowledged. Tenofovir was provided by Gilead Sciences and the gel was manufactured and supplied for the CAPRISA 004 trial by CONRAD. We thank Hua-Xin Liao, M. Anthony Moody, and Barton Haynes (Duke Human Vaccine Institute (DHVI) for HIV-1 mAb and antigen reagents and Glenn Overman (DHVI) for expert technical assistance in IgG depletion of plasma and CVL samples. Over the last decade, the CAPRISA 002 study team has received support from the National Institute of Allergy and infectious Disease (NIAID), the National Institutes of Health (NIH) (grants \# Al51794, \#Al104387, \#Al115981, \#Al116086), from CONRAD (USAID cooperative grant \#GP00-08-00005-00, subproject agreement \# PPA-09046), from the National Research Foundation (grant \# 67385), the Medical Research Council of South Africa, the Technology Innovation Agency, and the Columbia University-Southern African Fogarty AIDS International Training and Research Program (AITRP) funded by the Fogarty International Center, NIH (grant \# D43TW00231). D.A. was funded through the Medical Research Council of South Africa Self-Initiated Grant (MRC SIR), and the National Research Foundation (NRF) of South Africa training funds to carry out this study. This work was also supported by the NIH NIAID HVTN 
Laboratory Center UM1AI068618 and the NIH NIAID Duke Center for AIDS Research Immunology Core P30 Al 64518.

\section{DISCLOSURE}

The authors declare no conflict of interest.

c 2016 Society for Mucosal Immunology

\section{REFERENCES}

1. Grant, R.M. etal. Preexposure chemoprophylaxis for HIV prevention in men who have sex with men. N. Engl. J. Med. 363, 2587-2599 (2010).

2. Baeten, J.M. et al. Antiretroviral prophylaxis for HIV prevention in heterosexual men and women. N. Engl. J. Med. 367, 399-410 (2012).

3. Molina, J.-M. et al. On Demand PrEP With Oral TDF-FTC in MSM: Results of the ANRS Ipergay Trial. In Conference on Retroviruses and Opportunistic Infections (CROI). Abstract 23LB, Seattle, Washington, 23-26 February 2015.

4. Abdool Karim, Q. et al. Effectiveness and safety of tenofovir gel, an antiretroviral microbicide, for the prevention of HIV infection in women. Science 329, 1168-1174 (2010).

5. Marrazzo, J.M. et al. Tenofovir-based preexposure prophylaxis for HIV infection among african women. N. Engl. J. Med. 372, 509-518 (2015).

6. McCormack, S. et al. Pragmatic Open-Label Randomised Trial of Preexposure Prophylaxis: The PROUD Study. In Conference on Retroviruses and Opportunistic Infections (CROI). Abstract 22LB. Seattle, Washington, 23-26 February 2015.

7. Cranage, M. et al. Prevention of SIV rectal transmission and priming of Tcell responses in macaques after local pre-exposure application of tenofovir gel. PLoS Med. 5, e157 (2008).

8. Mureithi, M.W. et al. Preservation HIV-1-specific IFN $\gamma+$ CD4 + T cell responses in breakthrough infections following exposure to Tenofovir Gel in the CAPRISA 004 microbicide trial. J. Acquir. Immune Defic. Syndr. 60, 124-127 (2012).

9. Curtis, K.A. et al. Delayed maturation of antibody avidity but not seroconversion in rhesus macaques infected with simian HIV during oral pre-exposure prophylaxis. J. Acquir. Immune Defic. Syndr. 57, 355-362 (2011).

10. Laeyendecker, O. et al. Antibody maturation in women who acquire HIV infection while using antiretroviral pre-exposure prophylaxis. J. Infect. Dis. 212, 754-759 (2015).

11. Hare, C.B. et al. Seroreversion in subjects receiving antiretroviral therapy during acute/early HIV infection. Clin. Infect. Dis. 42, 700-708 (2006).

12. O'Sullivan, C.E. et al. Epstein-Barr virus and human immunodeficiency virus serological responses and viral burdens in HIV-infected patients treated with HAART. J. Med. Virol. 67, 320-326 (2002).

13. Payne, $\mathrm{H}$. et al. Reactivity of routine HIV antibody tests in children who initiated antiretroviral therapy in early infancy as part of the Children with HIV Early Antiretroviral Therapy (CHER) trial: a retrospective analysis. Lancet Infect. Dis. 15, 803-809 (2015).

14. Devito, C. et al. Mucosal and plasma IgA from HIV-1-exposed uninfected individuals inhibit HIV-1 transcytosis across human epithelial cells. J. Immunol. 165, 5170-5176 (2000).

15. Belyakov, I.M., Ahlers, J.D. \& Berzofsky, J.A. Mucosal AIDS vaccines: current status and future directions. Exp. Rev. Vaccines 3, S65-S73 (2004).

16. Smith, A.J. et al. Live simian immunodeficiency virus vaccine correlate of protection: immune complex-inhibitory fc receptor interactions that reduce target cell availability. J. Immunol. 193, 3126-3133 (2014).

17. Li, Q. et al. Live simian immunodeficiency virus vaccine correlate of protection: local antibody production and concentration on the path of virus entry. J. Immunol. 193, 3113-3125 (2014).

18. Tudor, D. et al. HIV-1 gp41-specific monoclonal mucosal IgAs derived from highly exposed but IgG-seronegative individuals block HIV-1 epithelial transcytosis and neutralize CD4(+) cell infection: an IgA gene and functional analysis. Mucosal Immunol. 2, 412-426 (2009).

19. Mazzoli, S. et al. HIV-specific mucosal and cellular immunity in HIV-seronegative partners of HIV-seropositive individuals. Nat. Med. 3 , 1250-1257 (1997).

20. Kaul, R. et al. Mucosal IgA in exposed, uninfected subjects: evidence for a role in protection against HIV infection. AIDS 15, 431-432 (2001).
21. Seaton, K.E. et al. HIV-1 specific IgA detected in vaginal secretions of HIV uninfected women participating in a microbicide trial in southern africa are primarily directed toward gp120 and gp140 specificities. PLoS One 9 , e101863 (2014)

22. Yates, N.L. et al. HIV-1 gp41 envelope IgA is frequently elicited after transmission but has an initial short response half-life. Mucosal Immunol. 6 , 692-703 (2013).

23. Lau, K.S. et al. Multi-scale in vivo systems analysis reveals the influence of immune cells on TNF- $\alpha$-induced apoptosis in the intestinal epithelium. PLOS Biol. 10, e1001393 (2012).

24. Janes, K.A. et al. A systems model of signaling identifies a molecular basis set for cytokine-induced apoptosis. Science 310, 1646-1653 (2005).

25. Woof, J.M. \& Mestecky, J. Mucosal immunoglobulins. Immunol. Rev. 206 64-82 (2005).

26. Russell, M.W. \& Mestecky, J. Humoral immune responses to microbial infections in the genital tract. Microbes Infect. 4, 667-677 (2002).

27. Devito, C. et al. Cross-clade HIV-1-specific neutralizing IgA in mucosal and systemic compartments of HIV-1-exposed, persistently seronegative subjects. J. Acquir. Immune Defic. Syndr. 30, 413-420 (2002).

28. Moja, P. et al. Prognostic value of antibodies to p66 during the course of HIV1 infection. Immunol. Lett. 56, 133 (1997).

29. French, M.A. et al. Isotype-switched immunoglobulin $\mathrm{G}$ antibodies to HIV Gag proteins may provide alternative or additional immune responses to 'protective' human leukocyte antigen-B alleles in HIV controllers. AIDS 27, 519-528 (2013).

30. Kersh, E.N. et al. Reduced inflammation and CD4 loss in acute SHIV infection during oral pre-exposure prophylaxis. J. Infect. Dis. 206, 770-779 (2012).

31. Hendrix, C.W. et al. MTN-001: randomized pharmacokinetic cross-over study comparing tenofovir vaginal gel and oral tablets in vaginal tissue and other compartments. PLoS One 8, e55013 (2013).

32. Louissaint, N.A. et al. Single dose pharmacokinetics of oral tenofovir in plasma, peripheral blood mononuclear cells, colonic tissue, and vaginal tissue. AIDS Res. Hum. Retroviruses 29, 1443-1450 (2013).

33. Kersh, E.N. et al. T cell chemo-vaccination effects after repeated mucosal SHIV exposures and oral pre-exposure prophylaxis. PLoS One 6, e19295 (2011).

34. Yates, N.L. et al. Vaccine-induced Env V1-V2 IgG3 correlates with lower HIV-1 infection risk and declines soon after vaccination. Sci. Transl. Med. 6, 228ra39 (2014).

35. Haynes, B.F. et al. Immune-correlates analysis of an HIV-1 vaccine efficacy trial. N. Engl. J. Med. 366, 1275-1286 (2012).

36. Zolla-Pazner, S. et al. Vaccine-induced lgG antibodies to V1V2 regions of multiple HIV-1 subtypes correlate with decreased risk of HIV-1 infection. PloS One 9, e87572 (2014).

37. Johansson, M. \& Lycke, N.Y. Immunology of the human genital tract. Curr. Opin. Infect. Dis. 16, 43-49 (2003).

38. Li, Z. et al. Transfer of IgG in the female genital tract by MHC class I-related neonatal $F c$ receptor $(\mathrm{FCRn})$ confers protective immunity to vaginal infection. Proc. Natl Acad. Sci. USA 108, 4388-4393 (2011).

39. Gupta, S. et al. The Neonatal Fc receptor (FcRn) enhances human immunodeficiency virus type 1 (HIV-1) transcytosis across epithelial cells. PLoS Pathog. 9, e1003776 (2013).

40. Ackerman, M.E. et al. Natural variation in FC glycosylation of HIV-specific antibodies impacts antiviral activity. J. Clin. Invest. 123, 2183-2192 (2013).

41. Zhou, M. \& Ruprecht, R.M. Are anti-HIV IgAs good guys or bad guys? Retrovirology 11, 109 (2014).

42. Sholukh, A.M. et al. Defense-in-depth by mucosally administered anti-HIV dimeric IgA2 and systemic IgG1 mAbs: Complete protection of rhesus monkeys from mucosal SHIV challenge. Vaccine 33, 2086-2095 (2015).

43. Nicholas, K.J. et al. B cell responses to HIV antigen are a potent correlate of viremia in HIV-1 infection and improve with PD-1 blockade. PLoS One 8, e84185 (2013).

44. Barouch, D.H. et al. Vaccine protection against acquisition of neutralization-resistant SIV challenges in rhesus monkeys. Nature 482, 89-93 (2012).

45. Wesemann, D.R. et al. Microbial colonization influences early B-lineage development in the gut lamina propria. Nature 501, 112-115 (2013). 
46. Anahtar, M.N. et al. Cervicovaginal bacteria are a major modulator of host inflammatory responses in the female genital tract. Immunity 42, 965-976 (2015).

47. Xiao, P. et al. Multiple vaccine-elicited nonneutralizing antienvelope antibody activities contribute to protective efficacy by reducing both acute and chronic viremia following simian/human immunodeficiency virus SHIV89.6P challenge in rhesus macaques. J. Virol. 84, 7161-7173 (2010).

48. Letvin, N.L. et al. Immune and genetic correlates of vaccine protection against mucosal infection by SIV in monkeys. Sci. Transl. Med. 3, 81 ra36 (2011).

49. Florese, R.H. et al. Contribution of nonneutralizing vaccine-elicited antibody activities to improved protective efficacy in rhesus macaques immunized with Tat/Env compared with multigenic vaccines. J. Immunol. 182, 3718-3727 (2009).

50. Gómez-Román, V.R. et al. Vaccine-elicited antibodies mediate antibodydependent cellular cytotoxicity correlated with significantly reduced acute viremia in rhesus macaques challenged with SIVmac251. J. Immunol. 174, 2185-2189 (2005).

51. Hessell, A.J. et al. Fc receptor but not complement binding is important in antibody protection against HIV. Nature 449, 101-104 (2007).

52. Barouch, D.H. et al. Protective efficacy of a global HIV-1 mosaic vaccine against heterologous SHIV challenges in rhesus monkeys. Cell 155, 531-539 (2013).

53. Bournazos, S. et al. Broadly neutralizing anti-HIV-1 antibodies require FC effector functions for in vivo activity. Cell 158, 1243-1253 (2014).

54. Bard, E. et al. Validation of a high sensitive immunoenzymatic assay to establish the origin of immunoglobulins in female genital secretions. J. Immunoassay Immunochem. 23, 145-162 (2002).

55. Mestecky, J., Alexander, R.C., Wei, Q. \& Moldoveanu, Z. Methods for evaluation of humoral immune responses in human genital tract secretions. Am. J. Reprod. Immunol. 65, 361-367 (2011).

56. Neutra, M.R. \& Kozlowski, P.A. Mucosal vaccines: the promise and the challenge. Nat. Rev. Immunol. 6, 148-158 (2006).

57. Devito, C. et al. Intranasal HIV-1-gp160-DNA/gp41 peptide prime-boost immunization regimen in mice results in long-term HIV-1 neutralizing humoral mucosal and systemic immunity. J. Immunol. 173, 7078-7089 (2004).

58. Duerr, A. Update on mucosal HIV vaccine vectors. Curr. Opin. HIV AIDS 5, 397-403 (2010).

59. Bebell, L.M. et al. Relationship between levels of inflammatory cytokines in the genital tract and CD4 + cell counts in women with acute HIV-1 infection. J. Infect. Dis. 198, 710-714 (2008).

60. Tomaras, G.D. et al. Initial B-cell responses to transmitted human immunodeficiency virus type 1: virion-binding immunoglobulin M (IgM) and IgG antibodies followed by plasma anti-gp41 antibodies with ineffective control of initial viremia. J. Virol. 82, 12449-12463 (2008).

61. Yates, N.L. et al. Multiple HIV-1-specific IgG3 responses decline during acute HIV-1: implications for detection of incident HIV infection. AIDS 25, 2089-2097 (2011).

62. Liao, H.-X. et al. Vaccine induction of antibodies against a structurally heterogeneous site of immune pressure within HIV-1 envelope protein variable regions 1 and 2. Immunity 38, 176-186 (2013).

63. Liao, H.-X. et al. Antigenicity and immunogenicity of transmitted/founder, consensus, and chronic envelope glycoproteins of human immunodeficiency virus type 1. J. Virol. 87, 4185-4201 (2013).

64. Gao, F. et al. Antigenicity and immunogenicity of a synthetic human immunodeficiency virus type 1 group m consensus envelope glycoprotein. J. Virol. 79, 1154-1163 (2005).

65. Gaschen, B. et al. Diversity considerations in HIV-1 vaccine selection. Science 296, 2354-2360 (2002).

66. Liao, H.-X. et al. A group M consensus envelope glycoprotein induces antibodies that neutralize subsets of subtype B and C HIV-1 primary viruses. Virology 353, 268-282 (2006).

67. Morris, L. et al. Isolation of a human anti-HIV gp41 membrane proximal region neutralizing antibody by antigen-specific single $B$ cell sorting. PLoS One 6, e23532 (2011).

68. Tibshirani, R. Regression Shrinkage and Selection via the Lasso. J. R. Stat. Soc. Ser. B 58, 267-288 (1996).

69. Lau, K.S. et al. In vivo systems analysis identifies spatial and temporal aspects of the modulation of TNF- $\alpha$-induced apoptosis and proliferation by MAPKs. Sci. Signal. 4, ra16 (2011). 